\title{
Régulation de débit d'un fluide dans un Laboratoire sur Puce
}

\author{
François Schwartz, Norbert Dumas, Abir Rezgui, Luc Hébrard \\ Pôle CNFM-MIGREST, Université de Strasbourg, France \\ francois.schwartz@unistra.fr, n.dumas@unistra.fr, rezgui@unistra.fr, luc.hebrard@unistra.fr
}

La formation Master à spécialité micro-nano électronique (MNE) de l'Université de Strasbourg propose, depuis l'année 2015, un enseignement intitulé «Projet de conception» présenté sous la forme d'un module de 4 heures de cours et de 24 heures de travaux pratiques. Les étudiants en seconde année de Master abordent le développement d'applications micro-fluidiques et plus précisément celui de l'électronique de pilotage d'actionneurs et de capteurs associés à un micro-laboratoire gravé sur une puce. Les généralités sur les régimes d'écoulement des fluides et le modèle du circuit fluidique y sont d'abord traitées. Une étude plus poussée conduit ensuite l'étudiant à dimensionner le circuit qui pilote une micropompe avec des niveaux de tensions spécifiques à la technologie High-Voltage $0,18 \mu \mathrm{m}$. Les notions d'automatique et de systèmes régulés sont indispensables en donnant un lien entre les objectifs et les performances attendues. Enfin, une série de simulations sous Cadence permet de relier les débits d'écoulement des fluides à la microélectronique de commande.

\section{Introduction}

Le parcours MNE du master Physique Appliquée et Ingénierie Physique de la Faculté de Physique et d'Ingénierie de l'Université de Strasbourg propose un enseignement de la microélectronique comme spécialité à ses étudiants de première et de seconde année. Une formation dans la conception des fonctions de l'électronique analogique et numérique y est dispensée et permet à nos étudiants de se spécialiser dans ce domaine. Afin d'étoffer l'offre de formation, de nouveaux modules d'enseignement ont été ajoutées et permettent aujourd'hui d'interagir avec d'autres disciplines.

A partir de financements obtenus notamment par le biais de la Coordination Nationale de la Formation en Microélectronique et en nanotechnologies (CNFM), l'acquisition d'outils performants nous permet à présent de créer des supports pédagogiques nouveaux et miniaturisés. Un circuit microfluidique comportant des canaux transportant des fluides (laboratoire sur puce) a été gravé (1) et embarque des micropompes pilotables à partir d'une électronique intégrée dans une puce. Cette maquette offre l'opportunité de mettre en évidence des phénomènes physiques à l'échelle nanoscopique et fait l'objet de cours et de séances de travaux pratiques. Un module d'enseignement a été mis en place à partir de l'année 2015 et donne un caractère hétérogène en mêlant les domaines de la fluidique, de l'électrostatique, de l'automatique et de la microélectronique. Il offre des champs nouveaux à enseigner dans le domaine de la modélisation des systèmes intégrés multiphysique et des technologies de la conception en microélectronique. C'est à partir d'un tel support qu'est enseigné le module de «Projet en conception » proposé aux étudiants de deuxième année MNE et de l'école TPS. 
Le système comprenant le microcircuit fluidique avec les micropompes et leur électronique de pilotage seront présentés respectivement dans les sections 2 et 3 . La séquence pédagogique d'enseignement sera déroulée dans la section 4 en insistant sur l'approche de conception. Enfin, une conclusion permettra de définir de nouvelles perspectives en vue de proposer des compléments à la séquence d'apprentissage en place.

\section{Présentation du système}

Le système est composé d'un circuit microfluidique destiné à faire circuler des réactifs et des échantillons à l'intérieur de différents canaux selon le procédé des laboratoires sur puce (Lab-On-Chip). Il s'agit d'un support de test de détection adapté à la présence d'un anticorps ou d'un antigène dans un échantillon. Le circuit fluidique possède des terminaisons de connexion à des flexibles sous la forme d'orifices, des canaux, une fenêtre de visualisation pour observer le fluide, et des mélangeurs afin de mélanger les échantillons et les réactifs introduits. La figure 1 donne un aperçu de la gravure des micro-canaux et des mélangeurs.

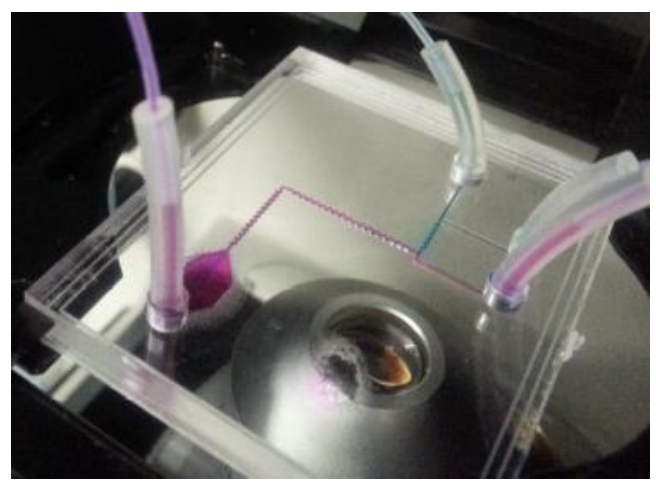

Fig.1. Le circuit microfluidique avec les mélangeurs.

Sur la figure 2 est représentée l'architecture du microcircuit complet avec trois micropompes pour activer la circulation des fluides injectés à travers chaque terminal de connexion (échantillon, enzyme, et substrat). Des portions de canaux sont ensuite constitués de motifs en serpentins et réalisent le mélange de ces fluides.

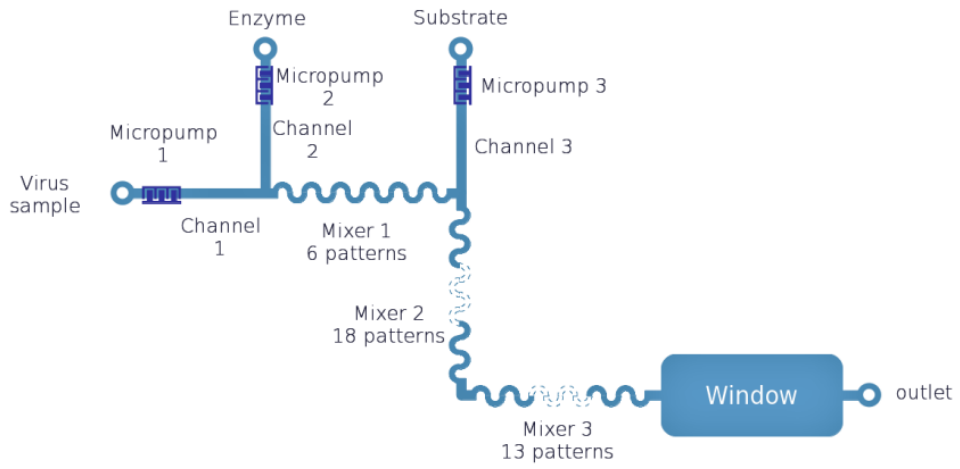

Fig.2. Représentation du micro-circuit étudié.

Les trois micro-pompes sont dimensionnées de façon identique et activent la circulation du fluide à partir d'électrodes entrelacées qui tapissent les parois du canal. L'interaction entre les électrodes et le fluide introduit à l'interface la formation d'une double couche électrique composée de charges immobiles très difficiles à déplacer pour 
la couche inférieure, et de charges libres sur la couche supérieure. Ce phénomène connu sous le nom d'électro-osmose $(2,3)$ peut être accentué par l'application d'un potentiel sur les électrodes. Le mécanisme d'entraînement des charges libres repose sur la présence d'un champ électrique tangentiel à la paroi du canal lié à la différence de potentiel entre les électrodes. Il est alors possible par une géométrie asymétrique des électrodes d'orienter le flot de charges libres davantage d'un côté du canal que de l'autre. Ce procédé est aujourd'hui exploité pour la circulation de fluides dans les microsystèmes et donne lieu à une large publication dans le domaine $(4,5)$. Un prototype de micropompe a été réalisé par nos soins à partir d'une machine $\mathrm{CNC}$ capable de graver des motifs jusqu'à une largeur de $100 \mu \mathrm{m}$. La figure 3 donne un aperçu de la réalisation avec notamment la présence de sept paires d'électrodes.

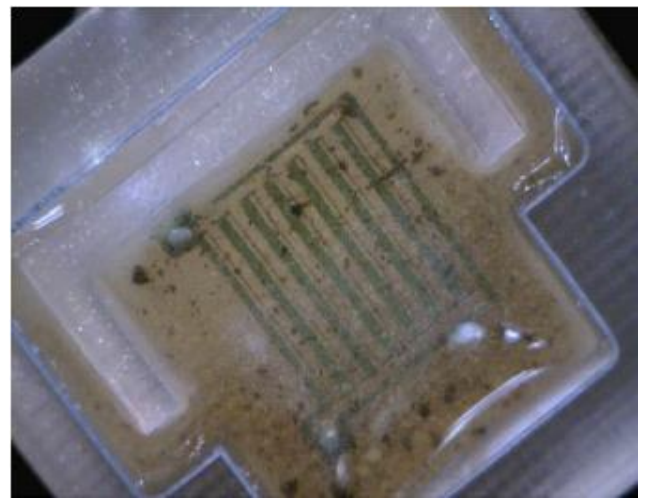

Fig.3. Prototype de la micro-pompe électro-osmotique.

Il apparaît que ce type de pompe présente un optimum de rendement pour une fréquence d'excitation du signal appliqué sur les électrodes. Le profil de vitesse d'entraînement du fluide présente en effet une résonance et toute la difficulté consiste à maintenir ce fonctionnement optimal sachant que la nature du fluide employé ou le vieillissement par corrosion provoque une dérive de cet optimum.

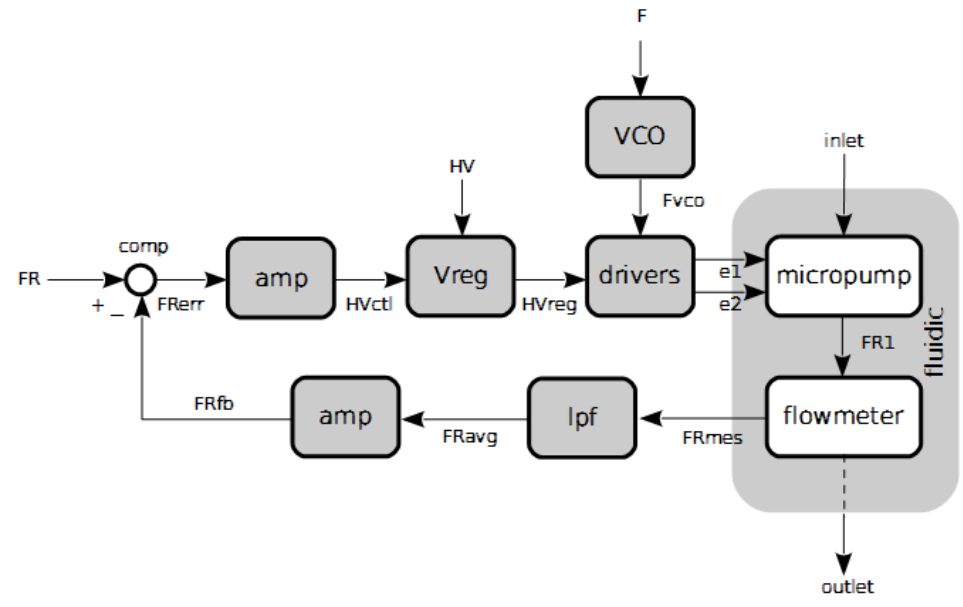

Fig.4. Boucle de régulation du débit du fluide.

Un capteur de débit est placé dans le circuit fluidique et une boucle de rétroaction est dimensionnée afin de réguler le débit du fluide. Cette structure doit permettre d'ajuster le débit du fluide en fonction de consignes précises tout en garantissant un rendement centré sur son optimum. 
La figure 4 présente les blocs du circuit microfluidique en blanc et les blocs de la chaine de régulation en gris. Le signal de consigne de débit -FR- est comparé au signal de retour de mesure du débit $-\mathrm{FR}_{\mathrm{fb}}$ - La chaîne de retour conforme le signal $-\mathrm{F}_{\mathrm{rmes}^{-}}$issu du débitmètre à l'aide d'un filtre passe-bas du second-ordre -lpf- et d'un amplificateur d'un gain de 100 -ampfb-. Le signal d'erreur $-\mathrm{F}_{\text {rerr- }}$, d'abord amplifié par l'amplificateur ampfw-, contrôle ensuite le régulateur en tension -Vreg- pour fournir au bloc -driversune tension ajustable en amplitude $-\mathrm{HV}_{\mathrm{dd}^{-}}$à partir du signal $\mathrm{HV}$ et dont le niveau permettra d'augmenter la vitesse et donc le débit du fluide. Ce niveau de tension peut atteindre plusieurs multiples de la tension d'alimentation de base du circuit et nécessite de faire l'appoint d'une pompe de charge non représentée sur le diagramme en blocs. Chaque micropompe est pilotée par le bloc -drivers- qui délivre une tension bipolaire d'amplitude $-\mathrm{HV}_{\mathrm{dd}}-\mathrm{au}$ rythme imposé par le $-\mathrm{V}_{\mathrm{CO}^{-}}$.

\section{III.L'électronique de commande}

Les blocs de la boucle de régulation et leur structure sont étudiés dans les détails avec une attention portée sur le circuit de pilotage des micropompes appelé -drivers-. Ce bloc doit fournir les niveaux de tension d'une amplitude suffisante afin de mettre en circulation les fluides au débit souhaité. Ces niveaux d'amplitude sont supérieurs à ceux rencontrés dans les technologies d'intégration dites «classiques » et nécessite l'utilisation d'une technologie adaptée. La technologie de process CMOS $0.18 \mu \mathrm{m}$ High-Voltage comprend une bibliothèque de composants capables de supporter des tensions pouvant aller jusqu'à $50 \mathrm{~V}$. Le bloc -drivers- effectue alors une translation entre les niveaux de tension d'amplitude $1,8 \mathrm{~V}$ du $-\mathrm{V}_{\mathrm{CO}}$ - et les niveaux de tension d'amplitude $10 \mathrm{~V}$ appliqués à la micropompe et fournis par la pompe de charge. La figure 5 donne le schéma d'un translateur de niveau (LevelShifter) structuré autour d'une paire différentielle polarisée en courant par le miroir de courant $\mathrm{M}_{\mathrm{b} 1}, \mathrm{M}_{\mathrm{b} 2}$, et $\mathrm{M}_{\mathrm{b} 3}$, et d'un étage de sortie dimensionné pour supporter des courants de charge efficaces de l'ordre de $30 \mathrm{~mA}$. La tension sur l'entrée in de niveau Low-Voltage $(1,8 \mathrm{~V})$ pilote les deux transistors $\mathrm{M}_{1}$ et $\mathrm{M}_{2}$ qui absorbent le différentiel $\mathrm{HV}_{\mathrm{dd}}\left(10 \mathrm{~V}\right.$ max.) et servent de tampon. Les transistors $\mathrm{M}_{3}, \mathrm{M}_{6}$, et $\mathrm{M}_{\mathrm{b} 3}$ sont dimensionnés de façon à recopier le différentiel de tension $\mathrm{V}_{\mathrm{GS}}$ du transistor $\mathrm{M}_{1}$ sur l'entrée grille-source du transistor de sortie $\mathrm{M}_{8}$ lorsque $\mathrm{M}_{2}$ est saturé.

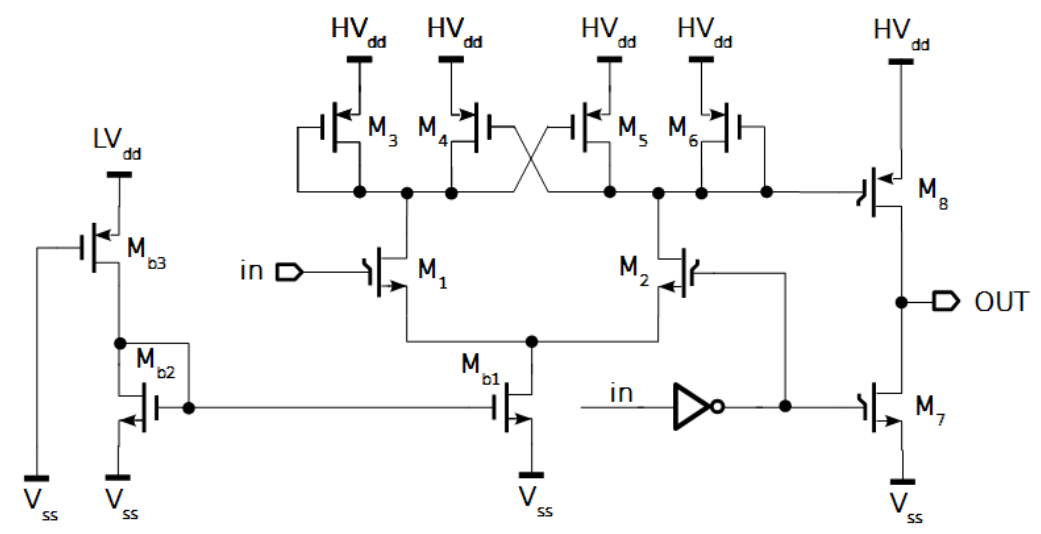

Fig.5. Schéma d'un bloc translateur de niveau présent dans le bloc -drivers- qui en compte deux pilotés de façon complémentaire.

Dans le cas où $\mathrm{M}_{2}$ est bloqué, les transistors $\mathrm{M}_{3}, \mathrm{M}_{4}, \mathrm{M}_{5}$, et $\mathrm{M}_{6}$, montés en miroir de tension replié, évitent de placer la grille du transistor $\mathrm{M}_{8}$ en état de haute impédance et 
forcent son potentiel à $\mathrm{H}_{\mathrm{vdd}}$. Le bloc -drivers- comporte deux structures translateur de ce type afin de délivrer une tension bipolaire aux bornes de chaque micropompe.

La figure 6 donne le layout de la puce dans laquelle est intégré le bloc -driversdéveloppé et fondu en technologie CMOS 0.18 HV. Le layout de la puce rassemble plusieurs projets réalisés par l'équipe du laboratoire ICube. Le schéma des autres blocs n'est pas détaillé durant cet enseignement et leur analyse se limite à leur caractérisation à partir d'un modèle comportemental de haut niveau développé en langage Verilog-A. Leur étude sera présentée dans la section 4 « Séquence pédagogique».
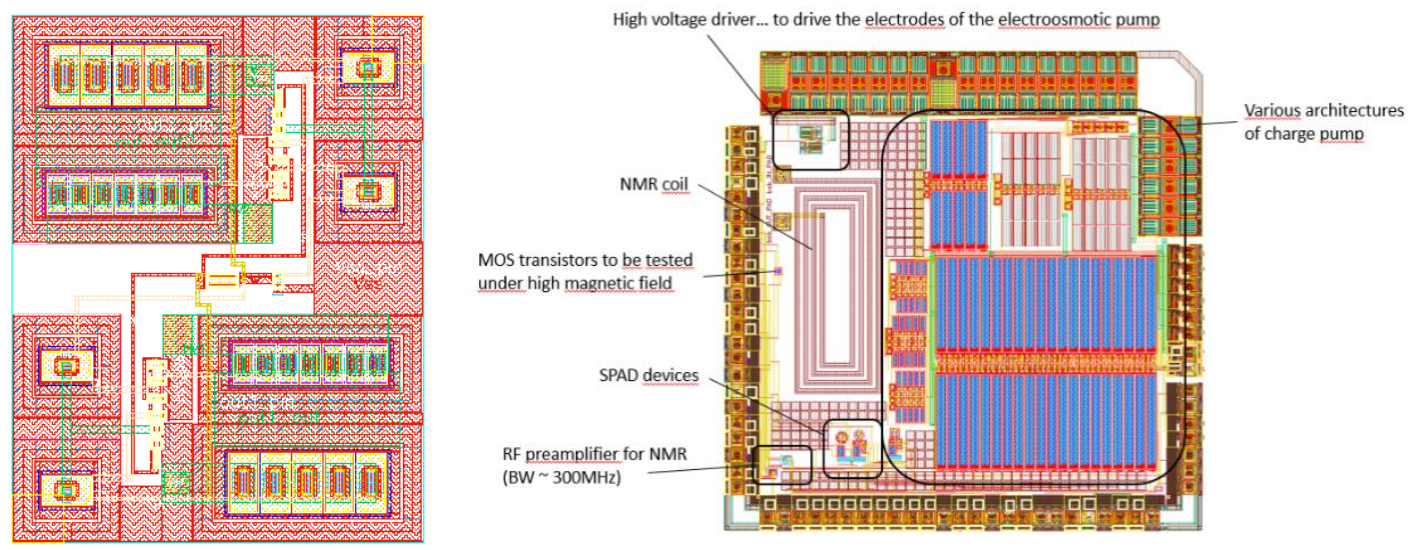

Fig.6. Layout du bloc -drivers- (à gauche) et de la puce (à droite)

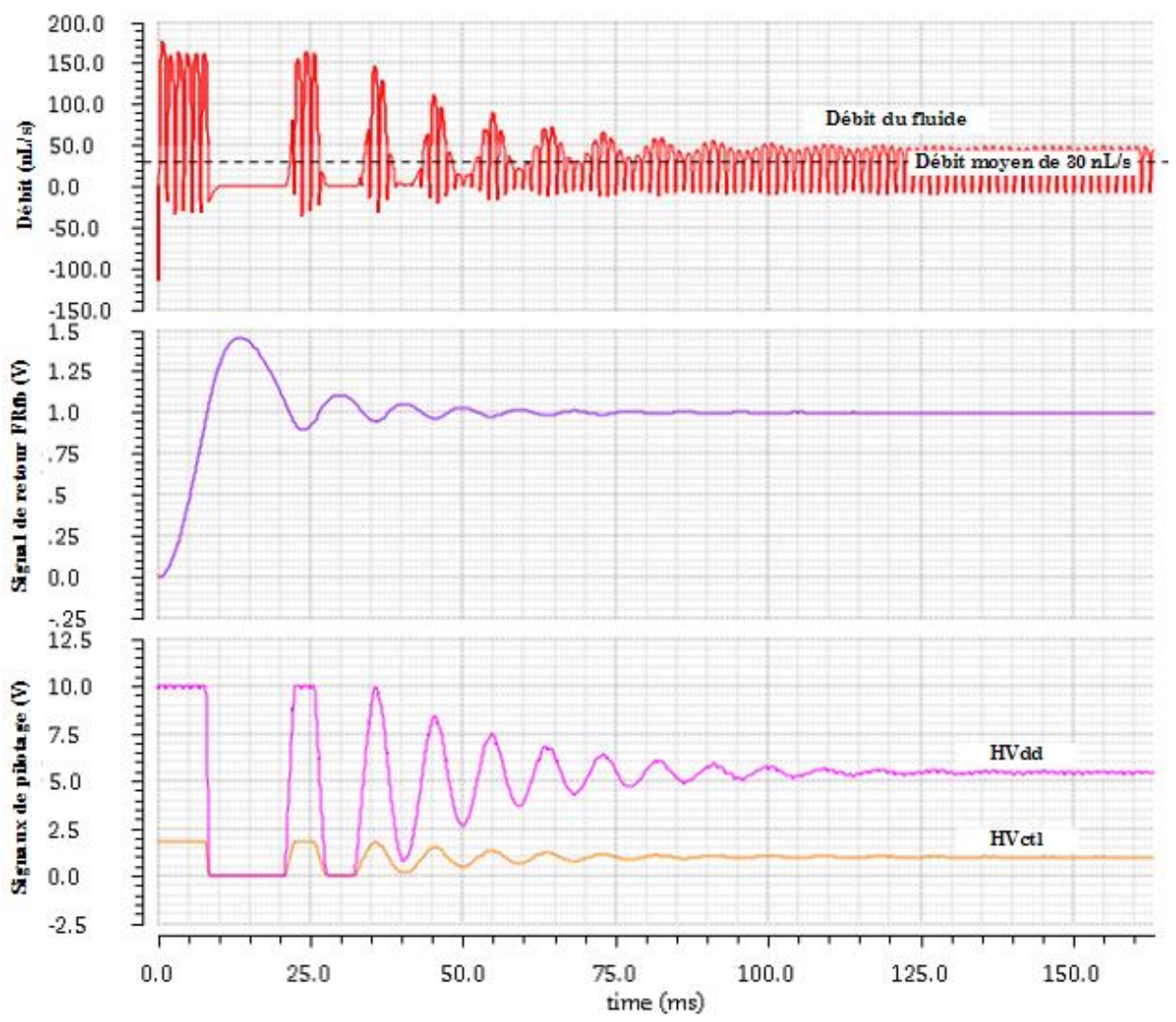

Fig.7. Réponse de la boucle de régulation à une consigne de débit de $30 \mathrm{~nL} / \mathrm{s}$ 


\section{IV.La séquence pédagogique}

La séquence d'enseignement est étalée sur un total de 4 heures de cours et 24 heures de travaux pratiques. Les heures de cours sont consacrées à la présentation du projet avec ses objectifs de conception. Parmi ces objectifs, le débit d'écoulement des fluides à l'intérieur des micro-canaux fixe la ligne directrice dans le dimensionnement des micropompes et de son électronique de commande associée. Une étude sur la dynamique des fluides est proposée avec pour finalité de développer un modèle de la micropompe. Cette étude conduit à un pré-dimensionnement des différents composants du microcircuit. Les diverses propriétés comme la vitesse, la pression, et le débit y sont pris en compte avec le phénomène d'électro-osmose comme vecteur d'écoulement du fluide. Le modèle de la micropompe est décrit en langage Verilog-A et prend en considération la répartition spatiale du champ électrique à travers le corps de la micropompe mais aussi la nature de l'écoulement (flot de Couette et de Poiseuille), la viscosité du fluide et ses caractéristiques telle que la conductivité. Un modèle équivalent d'impédance est également développé et donne le lien entre le domaine des fluides et celui de l'électricité. Il est alors possible de relier la tension de commande des micropompes électroosmotiques aux grandeurs de pression et de débit. La figure 6 illustre le modèle du microcircuit sous la forme d'un réseau de résistances hydrauliques soumises à des débits $\mathrm{Q}$ et des pressions $\mathrm{P}$ dans chaque portion du microcircuit. Chacune des trois micropompes est animée d'une vitesse de fluide uEO et impose une pression à la source laquelle fait converger les fluides introduits jusqu'à la fenêtre de visualisation caractérisée par une résistance hydraulique RWND. Chaque élément du microcircuit est dimensionné par une hauteur de canal $\mathrm{H}$ et d'une largeur $\mathrm{W}$. Une simulation sous Cadence permet ensuite d'ajuster ces dimensions par rapport aux performances de débit attendues.
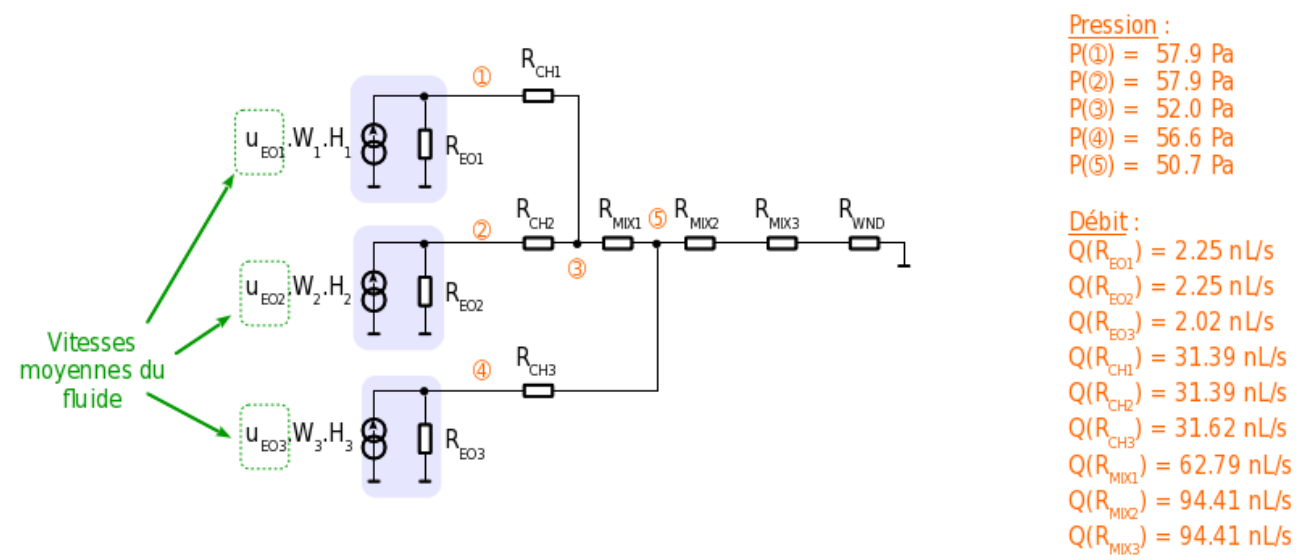

Fig.8. Modèle hydraulique du microcircuit fluidique avec ses trois micro-pompes. Chaque élément du microcircuit (canal, mélangeur, et fenêtre de visualisation) est modélisé par une résistance hydraulique.

A partir du modèle fluidique du microcircuit, l'électronique de commande est dimensionnée selon une approche Top-Down. L'étudiant analyse le dispositif de régulation à partir d'une description purement comportementale. Les différentes fonctions du circuit de pilotage sont dans un premier temps décrites à l'aide du langage Verilog-A. On introduit ensuite progressivement, pour chacune des fonctions, un modèle de description tenant compte de la technologie d'intégration (modèle de type Spectre) en vue schématique. 
L'étude suit la progression suivante :

- description comportementale en langage Verilog-A et simulation de chaque bloc du système ;

- simulation globale du système modélisé en langage Verilog-A;

- description sous forme de netlist et simulation du bloc -drivers- ;

- simulation globale du système modélisé en langage Verilog-A avec le bloc drivers- décrit par sa netlist;

- description sous forme de masques (layout) et simulation du bloc -drivers-;

- simulation globale du système modélisé en langage Verilog-A avec le bloc drivers- décrit par son layout.

Les notions d'automatique avec la minimisation du taux d'erreur en régime permanent ou de temps de réponse à $5 \%$ sont pris en considération avec à la clé un réglage en gain des blocs de la chaine de régulation. Sur la figure 7 est donné un scénario de régulation pour une consigne de débit moyen fixée à $30 \mathrm{~nL} / \mathrm{S}$ en sortie de chaque micro-pompe. Le débit est dans un premier temps oscillatoire-amorti puis stabilisé après une durée de 125 ms. A noter les niveaux d'amplitude du signal $\mathrm{HV}_{\text {ctl }}$ à l'entrée du bloc de régulation $-\mathrm{V}_{\text {reg- }}$ qui ne dépasse pas la tension de $1,8 \mathrm{~V}$ et $\mathrm{Hv}_{\mathrm{dd}}$ en sortie du bloc -drivers- avec un potentiel de l'ordre de $5 \mathrm{~V}$ appliqué ensuite à l'une des électrodes de la micro-pompe.

Enfin, la technologie High Voltage est largement étudiée avec une présentation des dispositifs à transistors MOS. Leurs limites de fonctionnement et leurs capacités à supporter des tensions supérieures aux tensions d'alimentation sont mis en relation avec leur empreinte layout. Les différents profils de transistors DMOS, LDMOS, DEMOS, isolés ou non, sont présentés ainsi que les techniques de cohabitation avec les zones dites de «basse-tension ».

Pour résumer, l'étudiant dispose de blocs décrits par leur comportement en langage Verilog-A qu'il caractérise séparément, qu'il assemble, et enfin simule. Partant d'une adéquation spécifications-performances, il progresse dans sa démarche de concepteur en introduisant des modèles de blocs orientés vers une représentation plus proche de la technologie sous la forme d'une description schématique. La séquence se ponctue par la prise du masque layout du bloc de commande -drivers- en tenant compte de règles de routage propre à la technologie CMOS $0.18 \mathrm{HV}$.

\section{Conclusion}

La séquence d'un nouvel enseignement intitulé «Projet de conception » a été présentée. Le flot de conception d'un cas concret de microsystème intégré est abordé par une modélisation et une simulation multi-domaines. Le microsystème étudié comprend une plaquette embarquant un microcircuit fluidique dans lequel des réactifs sont mélangés puis analysés ainsi qu'un dispositif micro-électronique destiné à la régulation de débit du fluide. La plaquette comprend également des micropompes à flux électroosmotique pilotées à partir d'un circuit supportant dans la technologie CMOS 0.18 HV des niveaux de tensions ici supérieurs à dix fois la tension d'alimentation.

L'étudiant met en pratique une démarche de conception descendante. Les modèles comportementaux du microcircuit fluidique et de ses micropompes électro-osmotiques sont complétés voire développés intégralement en langage Verilog-A. Des simulations sous Cadence permettent d'ajuster les dimensions des éléments du microcircuit fluidique sous les contraintes de spécifications en termes de vitesse d'écoulement et de débit. Une analyse du dispositif de régulation de débit est ensuite menée en développant le modèle comportemental de chaque bloc du dispositif. Les modèles sont alors affinés en tenant 
compte de la technologie d'intégration et en veillant à respecter les spécifications qui ont été propagées jusqu'au niveau du circuit. Finalement, le layout du bloc dédié au pilotage des micro-pompes est dessiné puis simulé.

La séquence présentée dans ce support pédagogique couvre les domaines de la microfluidique et de la microélectronique. De nouvelles perspectives d'amélioration sont envisageables pour les deux domaines. Le procédé de fabrication utilisé pour les micropompes s'appuie sur une conception par gravure facile, rapide, et peu onéreuse. Les performances observées en qualité de vitesse d'écoulement peuvent néanmoins être améliorées à partir d'une gravure en salle blanche qui offre une réduction d'échelle dans la pose des électrodes. De telles échelles permettent de multiplier l'intensité du champ électrique et donc la vitesse du fluide. L'emploi de matériaux plus résistants et moins sujets à la corrosion sont également envisageables. Dans le cas du dispositif de régulation, l'emploi des techniques neuronales sont envisagées dans le but de garantir un rendement de débit optimal en fonction des aléas de vieillissement des matériaux ou de la nature du fluide injecté. Un réseau de neurones peut en effet parfaitement s'adapter à l'usure du microcircuit fluidique en pilotant les niveaux d'amplitude appliqués aux micropompes ou encore en modifiant la fréquence délivrée par le VCO. Une telle étude est par ailleurs en cours et donnera matière à de nouveaux enseignements.

\section{Références}

1. A. Rezgui, M. Madec, N. Dumas, S. Guiton, Ch. Lallement, L. Hébrard, J. Haiech, F. Rufi, «Environnement de modélisation et de simulation pour la conception des laboratoires sur puces », Université de Strasbourg: Télécom Physique Strasbourg, Boulevard Sébastien Brandt, 67412 Illkirch.

2. RomenaSabur, M. A. Matin, "Study of Electro-osmotic flow in microfluidic devices", 2006, 3rd IEEE/EMBS International Summer School on Medical Devices and Biosensors.

3. Xiayan Wang, Chang Cheng, Shili Wang, and Shaorong Liu, "Electroosmotic pumps and their applications in microfluidic systems", Microfluid Nanofluidics. 2009 Feb 1; 6(2): 145.

4. M. Madec, Y. Gendrault, Ch. Lallement, J. Haiech, «Introduction à la biologie synthétique et au développement d'outils de conception dédiés », Université de Strasbourg : Télécom Physique Strasbourg, Boulevard Sébastien Brandt, 67412 Illkirch.

5. A.B.D. Brown, C. G. Smith, A.R. Rennie, «Pumping of water with ac electric fields applied to asymmetric pairs of microelectrodes », Physical Review E, Vol. 63, 0116305.

6. L. Ribetto, «Microfabricated All-Around-Electrode AC Electro-osmotic Micropmup », École Polytechnique Fédérale de Lausanne (EPFL), thèse présentée le 23 mars 2012. 